tecture Notes in Computational Vision and Biomechanics 34

João Manuel R. S. Tavares

Renato Manuel Natal Jorge Editors

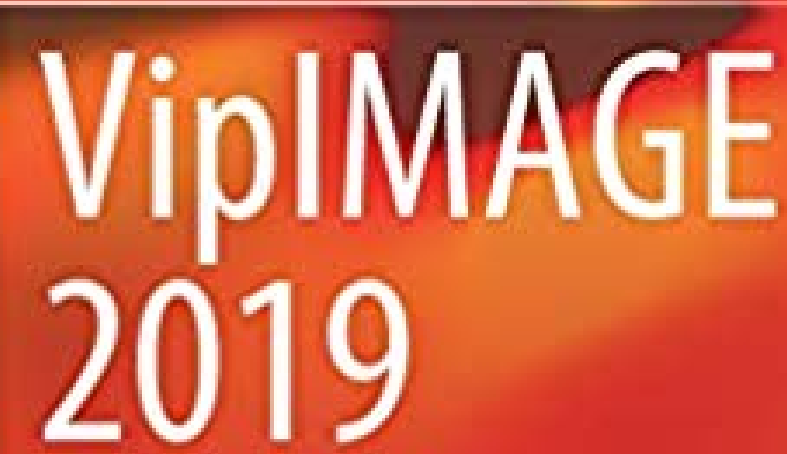

Proceedings of the VII ECCOMAS

Thematic Conference on Computational

Vision and Medical Image Processing,

October 16-18, 2019, Porto, Portugal

Q Springer 


\title{
Ultrasound Speckle Noise Reduction by Radio-frequency Data Filtering
}

\author{
Verónica Santo and Fernando C. Monteiro
}

\begin{abstract}
Ultrasound is a commonly used imaging modality for the examination of several pathologies due to its non-invasiveness, affordability and easiness of use. However, ultrasound images are degraded by an intrinsic artefact called 'speckle', which is the result of the constructive and destructive coherent summation of the ultrasound echoes. This paper aims to generate B-mode images out of radio-frequency (RF) data following standard procedures, a series of steps such as envelope detection, log-compression and scan conversion. Some low pass filters will be applied to RF data in order to achieve B-mode images with high quality by speckle noise reduction.
\end{abstract}

\section{Introduction}

Ultrasound imaging is one of the most important and cheapest instrument used for diagnostic purpose. However, the images obtained through this type of examination presents a characteristic noise type, known as speckle noise, which makes it difficult to analyse and diagnose $[1,2,3]$. Speckle noise is defined as multiplicative noise with a granular pattern formed due to coherent processing of backscattered signals from multiple distributed targets.

Over the last decades, several despeckling filters have been developed to reduce the speckle noise inherently present in ultrasound images without losing the diagnostic information. Image filtering techniques include adaptive filters, anisotropic diffusion and wavelets [2]. However, it is important to refer that the majority of the methods

Verónica Santo

Polytechnic Institute of Bragança, Campus Santa Apolónia, Bragança, Portugal, e-mail: veronica.ne.santo@gmail.com

Fernando C. Monteiro

Research Centre in Digitalization and Intelligent Robotics (CeDRI), Portugal

Polytechnic Institute of Bragança, Campus Santa Apolónia, Bragança, Portugal e-mail: monteiro@ipb.pt 
are applied to the B-mode images without taking advantage of the process used to obtaining these images from the RF data.

The RF signal is not usually available in the common ultrasound equipment and it is usually pre-processed (filtered and compressed) to improve its visualization. Compression is needed to reduce the dynamic range of the RF signal in order to adapted it to the dynamic range of the monitor [4].

Unfortunately, this pre-processing modifies the distribution of the RF signal. This step depends on a set of parameters such as the brightness, contrast, zoom and dynamic gain.

Recently, new types of filters have been proposed to remove speckle noise from RF data. In [5], the authors used a low pass frequency-shift, followed by a least mean square adaptive filter. Al-Asad [6] proposed a Short Time Fourier transform applied to the envelope of each RF line before reconstruction, followed by its application to the lateral dimension of the 2D image after reconstruction.

In this paper we intend to filter the raw RF data in order to reduce noise and limit the signal to the working bandwidth. This will be done by the application of denoising filters individually to the one-dimensional RF envelopes that will constitute the Bmode image. By filtering RF data in the process of B-mode image construction, we expect to obtain images with less speckle noise.

\section{From RF data to B-mode images}

The interaction of an acoustic wave with different tissue regions can be modelled by the backscattered radio-frequency (RF) signal. The ultrasound waves propagate through the tissue scattering and reflecting where variations in tissue density and elasticity occurs. Some of this energy returns to the transducer and it is recorded as a RF signal, as shown in Fig. 1.

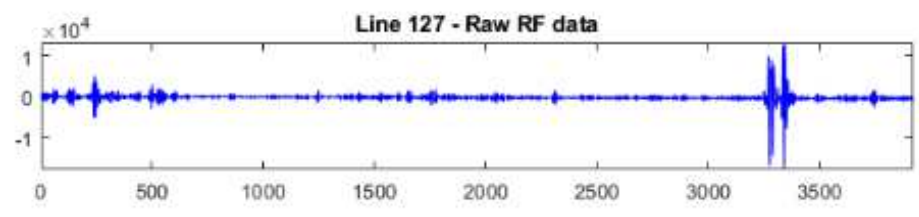

Fig. 1 Raw radio-frequency signal

There are several approaches for which useful information can be extracted from the RF signal to obtain B-mode ultrasound images. An overview of the various statistical distributions for modelling the envelope-detected RF signal can be found in [7]. 
To obtain an ultrasound B-mode image, the RF data goes through three signal processing phases, as shown in Fig. 2: IQ Demodulation, Envelope Detection, and Log compression.

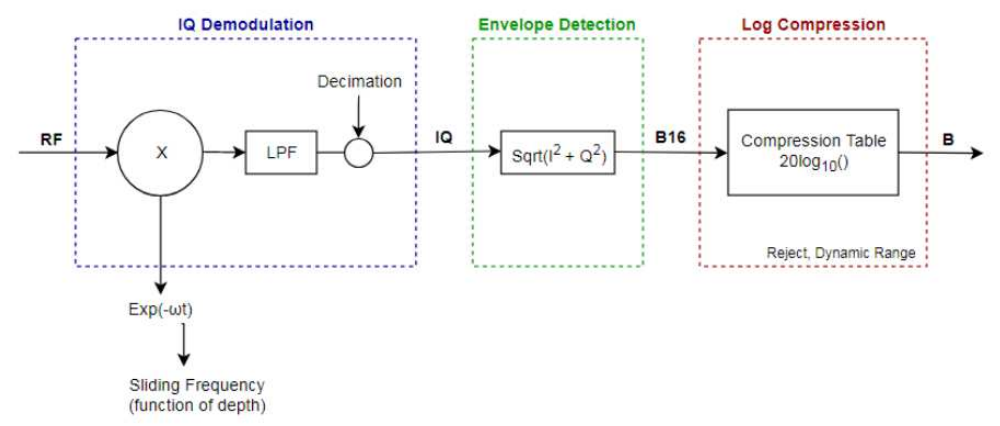

Fig. 2 From RF data to B-mode processing steps.

\subsection{IQ Demodulation}

In-phase Quadrature (IQ) demodulation the complex signal is low-pass filtered to remove the negative frequency spectrum and noise outside the desired bandwidth. The low-pass filter on the complex signal can be thought of as a filter applied to the real and imaginary part separately. With careful choice of low-pass filter, the remaining signal becomes weak for frequencies outside the pass-band for both components. This method multiplies an in-phase and quadrature-phase sinusoid with the input signal, causing signal content of that frequency to be accentuated and all other content to be reduced. The filter removes the frequencies stemming from the negative spectrum of the real RF signal, and the filter removes approximately half of the energy in the signal.

Decimation allows to reduce the sampling rate of a discrete-time signal. The Nyquist theorem states that the sampling frequency can be reduced to twice the cutoff frequency of the filter without loss of information. Low sampling rate reduces storage and computation requirements.

\subsection{Envelope Detection}

Since only the amplitude of the signal is of interest and not the frequency information itself, the envelope of the signal is calculated. The envelope is a non negative curve that connects the peaks (negative peaks are inverted) of the RF signal. Absolute and Hilbert transforms are commonly used methods to envelope detection. It returns the absolute or Hilbert amplitude variation of the amplitude of a time wave. 


\subsection{Logarithmic compression}

In order to obtain a 2D image, a series of adjacent beams (RF signals) are recorded from which a RF matrix is built. This RF matrix is then further processed, ultimately leading to the B-mode image, which is visualized on the screen.

To visualize the envelope signal of a scan line the value of each sample point is mapped to a 8 bit greyscale colour map. The result of the compression is a first B-mode image, each column of pixels corresponding to a scan line. In order to improve the contrast of the image the brightness values of the B-mode image have to be adjusted to the dynamic range of the human eye. Usually, non-linear mapping functions are used, which keep the order of the values but reduce the difference between high peaks in the signal and lower values and therefore increasing the overall brightness of the image.

The compressed image most likely contains various image artefacts and speckles. In order to improve the image quality scan conversion has to be performed. The RF data contains the single scan lines as single columns (See Fig. 3). Since these scan lines are usually not parallel they have to be arranged geometrically in the final image depending on the geometry of the probe as fan shaped images, as shown in Fig. 3.

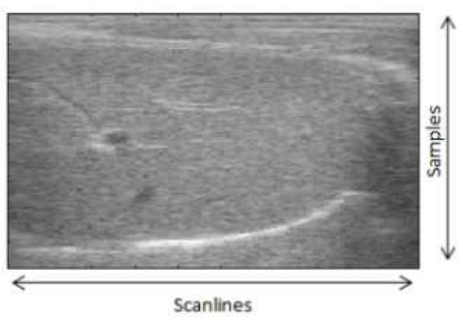

Fig. 3 Logarithmic conversion

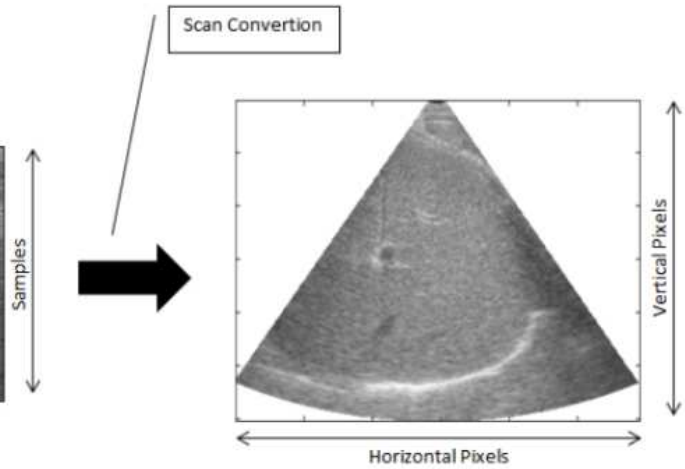

\section{Radio-frequency Filtering Methods}

Usually, techniques of image enhancement and speckle noise reduction are applied on B-mode ultrasound image [2], after the RF data goes through the phases mentioned previously.

In this work, we propose to apply several low-pass filters (LPF) before the RF signals turn into the B-mode image. In order to filter the RF signal we need to focus on the first ultrasound signal processing technique, the IQ Demodulation. In this phase it is possible to apply several LPF and test different decimation values. 


\subsection{Low-pass Filters}

Attenuate or reduce the amplitude of the frequencies larger than the cut-off frequency where excessive phase shift may cause oscillations. The amount of attenuation for each frequency varies from filter to filter. The different filters with different frequencies are used to remove noise. We have designed 3 classic analogue filter types to filter the noise in RF sign.

- Bessel filter: A Bessel filter is a type of analogue linear filter used within RF that has a maximally flat group or phase delay. Bessel low-pass filters, therefore, provide an optimum square-wave transmission behaviour. This preserves a wave shape of signals within the pass-band. However, the pass-band gain of a Bessel low-pass filter is not as flat as that of the Butterworth low-pass, and the transition from pass-band to stop-band is by far not as sharp as that of a Chebyshev low-pass filter.

- Butterworth filter: Butterworth filters have a maximally flat response. That means all the derivatives of the amplitude with frequency are zero at DC. The Butterworth response is a good compromise between attenuation characteristic and group delay.

- Chebyshev filter: The Chebyshev filter has ripples in the pass-band and the value of the ripples is a parameter that is selected as part of the filter design.

\section{Results and Analysis}

Figure 4 shows the results obtained by using the Bessel, Butterworth and Chebyshev filters, with different decimation of 1 and 30, and absolute and Hilbert envelopes.

By comparing the images obtained and the corresponding signal representation, we can observe that the Chebyshev filter is the one with the best results in speckle noise reduction, when used with the Hilbert Envelope. Increasing decimation from 1 to 30 produces an improvement in the results, however, for values greater than 30 an undesirable image distortion or blurring effect happens.

In order to support the visual analysis we need quantitative evaluation data. For that we evaluated speckle noise reduction with some state-of-the-art quality measures: mean square error (MSR), square root of the mean square error (RMSR), the peak-signal-to-noise radio (PSNR), signal to noise ratio (SNR), quality index and structural similarity index (MSSIM). Table 1 shows the results obtained.

From Table 1 we can see that the combination of Chebyshev filter with decimation of 30 and Hilbert envelope produces the best result in speckle noise reduction in all used quality measures. When compared to Bessel and Butterworth filter, a Chebyshev filter can achieve a sharper transition between the pass band and the stop band with a lower order filter. The sharp transition between the pass-band and the stop-band of a Chebyshev filter-produces smaller absolute errors and faster execution speeds than 

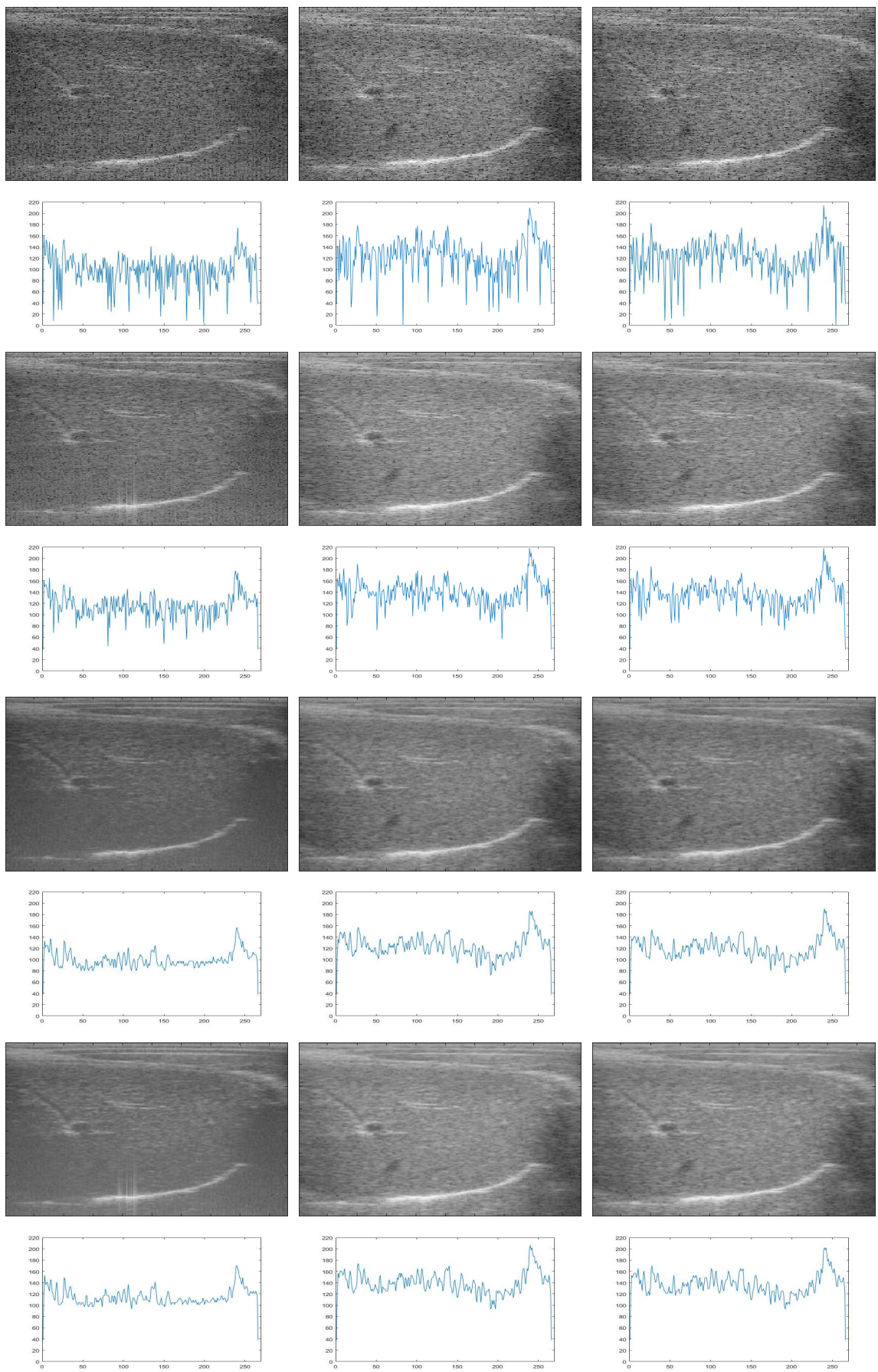

Bessel

Butterworth

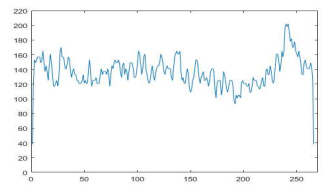

Chebyshev

Fig. 4 Influence of Bessel, Butterworth and Chebyshev filters with different decimation and envelope. Below each result we have the profile extracted from the column 137 after denoising. First row shows the results with decimation 1 and Absolute envelope. Second row shows the results with decimation 1 and Hilbert envelope. Third row shows the results with decimation 30 and Absolute envelope. Fourth row shows the results with decimation 30 and Hilbert envelope. 
Table 1 Speckling reduction according to the performance computed with state-of-the-art evaluation metrics. First six rows use downsampling $=1$ and the last six rows use downsampling $=$ 30 .

\begin{tabular}{lccccccc}
\hline Filter & Envelope & MSR & RMSE & PSNR & SNR & Quality & MSSIM \\
\hline Bessel & Absolute & 2145.09 & 46.32 & 17.83 & 11.20 & 0.03 & 0.10 \\
Butterwoth & Absolute & 954.19 & 30.89 & 21.34 & 15.37 & 0.10 & 0.15 \\
Chebyshev & Absolute & 1030.41 & 32.10 & 21.01 & 14.94 & 0.15 & 0.19 \\
Bessel & Hilbert & 950.79 & 30.83 & 21.36 & 15.12 & 0.05 & 0.18 \\
Butterwoth & Hilbert & 308.62 & 17.57 & 26.25 & 20.71 & 0.19 & 0.29 \\
Chebyshev & Hilbert & 279.38 & 16.71 & 26.68 & 21.04 & 0.29 & 0.37 \\
Bessel & Absolute & 1526.20 & 39.07 & 19.31 & 12.57 & 0.46 & 0.65 \\
Butterwoth & Absolute & 270.12 & 16.43 & 26.83 & 20.77 & 0.84 & 0.88 \\
Chebyshev & Absolute & 367.89 & 19.18 & 25.48 & 19.32 & 0.91 & 0.93 \\
Bessel & Hilbert & 643.17 & 25.36 & 23.06 & 16.79 & 0.52 & 0.71 \\
Butterwoth & Hilbert & 21.08 & 4.59 & 37.90 & 32.35 & 0.88 & 0.91 \\
Chebyshev & Hilbert & $\mathbf{1 7 . 6 1}$ & $\mathbf{4 . 2 0}$ & $\mathbf{3 8 . 6 8}$ & $\mathbf{3 3 . 0 3}$ & $\mathbf{0 . 9 5}$ & $\mathbf{0 . 9 6}$
\end{tabular}

Bessel and Butterworth filters. Chebyshev filter is used where the frequency content of a signal is more important than having a constant amplitude.

Figure 5 shows the B-mode image obtained with pre-processing, without filtering; and B-mode image obtained with pre-processing and noise filtering. It is possible to observe that in the filtered $\mathrm{Rf}$ image the structures present in the ultrasound shows more cleraly, which allows to analyze the image without losing the diagnostic information.

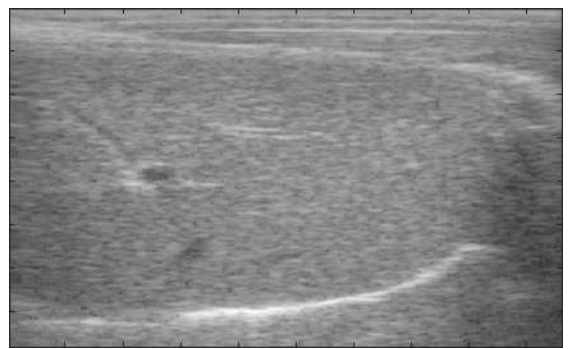

(a) Filtered

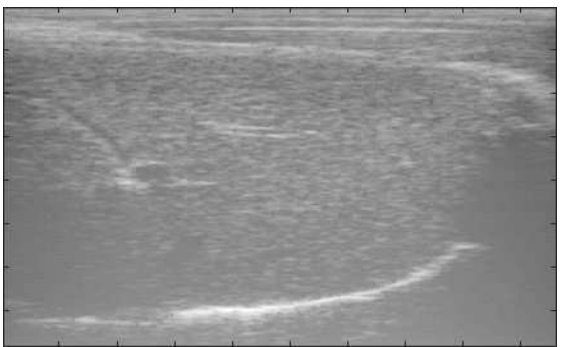

(b) Non Filtered

Fig. 5 B-mode images with and without filtering. 


\section{Conclusion}

In this paper we proposed and approach to reduce speckle noise in ultrasound images by filtering the raw RF data, before obtain the B-mode image. This was done by using denoising filters individually to the $1 \mathrm{D}$ RF envelopes that will constitute the B-mode image. In the IQ demodulation phase we tested different low pass filters: Bessel, Butterworth and Chebyshev, with different decimation values and different envelope techniques. To test the effectiveness of the speckle noise reduction we evaluate the results with several state-of-the-art quality measures. From the obtained evaluation results we can conclude that filtering in RF mode, before the conversion to B-mode, is an effective method to reduce the speckle noise.

\section{References}

1. Ortiz, S.H.C., Chiu, T., Fox, M.D.: Ultrasound image enhancement: A review. Biomedical Signal Processing and Control. 7(5), 419-428 (2012)

2. Monteiro, F.C., Rufino, J., Cadavez, V.: Towards a comprehensive evaluation of ultrasound speckle reduction. Lecture Notes in Computer Science, vol. 8814, 141--149 (2014)

3. Joel, T. Sivakumar, R.: Despeckling of Ultrasound Medical Images: A Survey. Journal of Image and Graphics, 1(3), 161-165 (2013)

4. Seabra, J., Sanches, J.: Modeling log-compressed ultrasound images for radio frequency signal recovery. Int. Conference of the IEEE Engineering in Medicine and Biology Society, p. 426-429, Vancouver, Canada (2008)

5. Wang, S., Li, C., Ding, M., Yuchi, M.: Frequency-shift low-pass filtering and least mean square adaptive filtering for ultrasound imaging. Progress in Biomedical Optics and Imaging Proceedings of SPIE, vol. 9790, p. 97900P (2016)

6. Al-Asad, J.F.: Despeckling the 2D medical ultrasound image through individual despeckling of the envelopes of its 1D radio frequency echo lines by STFT. Journal Image Graphics. vol. 4, 67--72 (2016).

7. Destrempes, F., Cloutier, G.: A critical review and uniformized representation of statistical distributions modeling the ultrasound echo envelope. Ultrasound in Medicine and Biology. 36(7), 1037-1051 (2010). 